\title{
THE CARATHÉODORY EXTENSION THEOREM FOR VECTOR VALUED MEASURES
}

\author{
JOSEPH KUPKA
}

\begin{abstract}
This paper comprises three advertisements for a known theorem which, the author believes, deserves the title of the Caratheodory extension theorem for vector valued premeasures. Principal among these is a short and transparent proof of Porcelli's criterion for the weak convergence of a sequence in the Banach space of bounded finitely additive complex measures defined on an arbitrary field, and equipped with the total variation norm. Also, a characterization of the so-called Caratheodory Extension Property is presented, and there is a brief discussion of the relevance of this material to stochastic integration.
\end{abstract}

Amongst the numerous criteria for the extendability of a vector valued premeasure (see [9] for an admirable summary and comprehensive bibliography), precisely one stands out both for its simplicity and for its applicability. Our aim in this paper is to rescue this criterion from its relative obscurity within the specialist literature by providing three "advertisements" for it. The main advertisement will be a short, transparent proof of Porcelli's characterization of the weak convergence of a sequence of finitely additive complex measures (Theorem 2). The second advertisement will be a simple characterization of those Banach spaces for which the classical Carathéodory extension theorem remains essentially valid (Theorem 5), and the third advertisement will be an indication of the usefulness of the criterion to the construction of stochastic integrals.

We begin with a brief discussion of the criterion itself. Recall that a (Banach space valued) set function $\mu$ is said to be strongly bounded if we have $\lim _{n \rightarrow \infty} \mu\left(E_{n}\right)=0$ for every infinite sequence $\left\{E_{n}\right\}$ of pairwise disjoint sets in the domain of $\mu$, and that $\mu$ is a premeasure if we have

$$
\mu(E)=\sum_{n} \mu\left(E_{n}\right)
$$

for every finite or infinite sequence $\left\{E_{n}\right\}$ of pairwise disjoint sets in the domain of $\mu$ whose union, $E$, also lies in the domain of $\mu$. Here now is the theorem which, we feel, deserves the title of the Carathéodory extension theorem for Banach space valued premeasures.

Received by the editors October 31, 1977.

AMS (MOS) subject classifications (1970). Primary 46E99, 46-02, 54A20, 46G10; Secondary $60 \mathrm{G} 05,60 \mathrm{H} 05$.

Key words and phrases. Vector valued premeasures, weak convergence of measures, Carathéodory Extension Property, stochastic integration. 
1. TheOREM. Let $\mathbf{F}$ be a field of subsets of a set $\Omega$, let $X$ be a Banach space, and let $\mu: \mathrm{F} \rightarrow X$ be a premeasure. Then $\mu$ may be uniquely extended to an $X$ valued measure $\mu^{*}$ on $\mathbf{A}$, the $\sigma$-field generated by $\mathbf{F}$, if and only if $\mu$ is strongly bounded on F. Moreover, if the total variation measure, $|\mu|$, of $\mu$ is finite, then we also have $|\mu|^{*}=\left|\mu^{*}\right|$.

The major portion of Theorem 1 was first obtained in greater generality by Sion [17] who was later [16] to generalize it even a bit further (to uniform semigroup valued premeasures). His arguments are elementary but exceedingly lengthy and technical. An equally elementary and much shorter approach to Theorem 1 as it stands is to construct a control measure for $\mu$ via the arguments of Traynor in [18, Theorem 1.3, p. 256ff]. This procedure reduces the construction of $\mu^{*}$ to the classical metric approximation argument of Găină [8]. Apart from the intrinsic appeal of this elementary approach, it also increases the power of Theorem 1. The Porcelli result (Theorem 2 below) was actually used in the original, nonelementary construction of a control measure in this setting [2].

Our first advertisement for Theorem 1 is a short proof of Porcelli's criterion for the weak convergence of a sequence in the space $\mathbf{M}_{f}=\mathbf{M}_{f}(\Omega, F)$ of bounded finitely additive complex measures defined on a field $\mathbf{F}$ of subsets of a set $\Omega$, and equipped with the total variation norm. Porcelli's original proof [15] was extremely long and gruelling, while the direct proof due to Darst [5] still contains many technical arguments. The present proof is less elementary, but is devoid of technicalities and directly relates the finitely additive result to its analogue for countably additive measures.

2. THEOREM. A sequence $\left\{\mu_{n}\right\}$ in $\mathbf{M}_{f}$ converges weakly in $\mathbf{M}_{f}$ if and only if the limit

$$
\lim _{n \rightarrow \infty} \sum_{j=1}^{\infty} \mu_{n}\left(F_{j}\right)
$$

exists for every sequence $\left\{F_{j}\right\}$ of pairwise disjoint sets in $\mathbf{F}$.

Proof. The necessity of the condition is trivial, and so we prove the sufficiency. In view of the Stone space construction, there is no loss of generality in assuming that the elements of $\mathbf{M}_{f}$ are in fact premeasures. It now follows from the second part of Theorem 1 that the map which sends each $\mu \in \mathbf{M}_{f}$ into its Carathéodory extension $\mu^{*}$ on $\mathbf{A}$, the $\sigma$-field generated by $\mathbf{F}$, determines an isometric isomorphism between $\mathbf{M}_{f}$ and the space $\mathbf{M}=$ $\mathbf{M}(\Omega, \mathbf{A})$ of countably additive complex measures defined on $\mathbf{A}$, and equipped with the total variation norm. It therefore suffices to show that the sequence $\left\{\mu_{n}^{*}\right\}$ converges weakly in $\mathbf{M}$, and in view of the classical results in this direction [7, Theorem 5, p. 308; Theorem 8, p. 309], it is sufficient only to show that the $\mu_{n}^{*}$ converge pointwise on $\mathbf{A}$.

To this end, we first conclude from the given assumption and from a slight 
modification to the argument in [4, Theorem 2, p. 1296] that the $\mu_{n}$ are uniformly strongly bounded. (This means simply that the limit which is asserted to exist in the definition of strong boundedness is uniform in $n$.) We next let $\mathbf{c}$ denote the Banach space of convergent sequences of complex numbers, equipped with the supremum norm, and we define a $\mathbf{c}$ valued "superpremeasure" $\mu$ on $\mathbf{F}$ by

$$
\boldsymbol{\mu}(F)=\left(\mu_{1}(F), \mu_{2}(F), \ldots\right)
$$

for all $F \in \mathbf{F}$. The fact that $\mu$ is a strongly bounded premeasure on $\mathbf{F}$ follows from the uniform strong boundedness of the $\mu_{n}$. Theorem 1 thus allows $\mu$ to be extended to a $c$ valued measure $\mu^{*}$ on $A$. From the uniqueness part of Theorem 1, we conclude easily that

$$
\mu^{*}(E)=\left(\mu_{1}^{*}(E), \mu_{2}^{*}(E), \ldots\right)
$$

for all $E \in \mathbf{A}$. But now the mere fact that $\mu^{*}$ assumes values in $\mathbf{c}$ gives the desired conclusion.

A portion of the above argument does not require the $\mu_{n}$ to be scalar valued. We isolate this portion into the following corollary, which generalizes [2, Theorem 4, p. 1001].

3. Corollary. Let $\mathbf{F}$ be a field of subsets of a set $\Omega$, let $\mathbf{A}$ be the $\sigma$-field generated by $F$, let $X$ be a Banach space, and let $\left\{\mu_{n}\right\}$ be a sequence of countably additive $X$ valued measures defined on $\mathrm{A}$. Then the limit $\lim _{n \rightarrow \infty} \mu_{n}(E)$ exists for all sets $E \in \mathbf{A}$ if and only if this limit exists precisely for those sets $E \in \mathbf{A}$ which constitute the union of a sequence $\left\{F_{j}\right\}$ of (pairwise disjoint) sets in $\mathbf{F}$.

A similar sort of application of Theorem 1 appears in [18, Theorem 3.4, p. 363].

Our second advertisement for Theorem 1 is a characterization of those Banach spaces for which the original Carathéodory extension theorem remains essentially valid.

4. Definition. A Banach space $X$ is said to possess the Carathéodory Extension Property if, for every field $\mathbf{F}$, every bounded $X$ valued premeasure defined on $\mathbf{F}$ extends (uniquely) to a countably additive $X$ valued measure defined on the $\sigma$-field generated by $\mathbf{F}$.

Simple examples (e.g. [9, Ex. 1, p. 176]) show that without the requirement that the premeasure be bounded, no Banach space at all will possess the Carathéodory Extension Property.

5. Theorem. The Banach space $X$ possesses the Carathéodory Extension Property if and only if $X$ does not contain an isomorphic copy of $\mathbf{c}_{0}$.

Proof. In the light of Theorem 1, the fact that a Banach space which does not contain $\mathbf{c}_{0}$ has the Carathéodory Extension Property is well known [6, Theorem 1.8, p. 216]. It remains, therefore, to produce a $c_{0}$ valued premeasure 
which is bounded but not strongly bounded (and hence not extendable).

To this end, let $\Omega=[0,1)$, let $F$ denote the field of finite disjoint unions of intervals of the form $[a, b) \subset \Omega$, let $t_{n}=n^{-1}$ for $n=1,2, \ldots$, and let $\delta_{n}$ denote point mass at $t_{n}$. The significance of $\mathbf{F}$ in relation to the $t_{n}$ lies in the fact that, for all $F \in \mathbf{F}$, we have $0 \in F$ if and only if $F$ contains all but finitely many of the $t_{n}$, while $0 \notin F$ if and only if $F$ contains at most finitely many of the $t_{n}$. It follows that if we define $\mu$ on $F$ by

$$
\mu(F)=\left(\delta_{2}(F)-\delta_{1}(F), \delta_{3}(F)-\delta_{2}(F), \ldots\right)
$$

for all $F \in \mathbf{F}$, then $\mu$ assumes values in $\mathbf{c}_{0}$ simply because all but finitely many coordinates of each value of $\mu$ vanish. Moreover, if a set $F \in \mathbf{F}$ is the union of a sequence $\left\{F_{j}\right\}$ of pairwise disjoint elements of $\mathbf{F}$, then we have $\mu\left(F_{j}\right)=0$ for all but finitely many $j$, so that the countable additivity of $\mu$ follows vacuously from the countable additivity of its coordinate measures. It is obvious that $\mu$ is bounded but not strongly bounded.

6. Corollary. Every weakly complete Banach space possesses the Carathéodory Extension Property.

Proof. This follows at once from the fact that every closed subspace of a weakly complete space is weakly complete, whereas $\mathbf{c}_{0}$ is not weakly complete.

Our third advertisement for Theorem 1 is to point out its relevance to the definition of stochastic integrals arising from a predetermined stochastic process. Beginning with the work of Pellaumail in [13], an attempt has been made to identify the stochastic integral as a special case of the classical Bartle integral with respect to a vector valued measure (see [1] and [11]). This identification might be called the "Lebesguification" of the stochastic integral, for it makes possible the same kind of advancement of the original theory as that made by the Lebesgue-Stieltjes integral over the RiemannStieltjes. Moreover, the identification is achieved in precisely the same way, i.e. the given stochastic process is (first) converted into a vector measure in precisely the same way that a monotone function is converted into a Lebesgue-Stieltjes measure. Thus an extension theorem is required for what we might term stochastic premeasures, and an examination of even the most recent literature reveals that many workers in this area are still laboring under some of the more difficult criteria for the extendability of vector valued premeasures (e.g., see [11, Theorem 5, p. 291] and [10, Theorem 10.10, p. 109]).

But more than this, stochastic premeasures assume their values exclusively in $L^{p}$ spaces (for $1 \leqslant p \leqslant \infty$ ). Now it is well known that when $p$ is finite these spaces are weakly complete [7, Corollary 2 , p. 288; Corollary 29, p. 69; Theorem 6, p. 290], so that, by Corollary 6, they possess the Carathéodory Extension Property. Even the more general $L_{E}^{p}$ spaces considered by Metivier in [12, p. 761ff] (wherein the question of extendability is conspicuously 
ignored!) are weakly complete under Metivier's assumption that the Banach space $E$ is reflexive (see [14, Theorem 5.7, p. 135] and [3, Corollary 2, p. 285]). So in nearly all cases (i.e. except when $p=\infty$ ) we conclude: If $\mu$ is a stochastic premeasure defined on a ring (most usually of subsets of $\mathbf{R}$ ), then in order to extend $\mu$ to the generated $\sigma$-field it is sufficient merely to establish that $\mu$ is bounded, and in order to extend $\mu$ to the generated $\delta$-ring it is sufficient merely to establish that $\mu$ is locally bounded (in the sense of [9, p. 177]).

\section{REFERENCES}

1. R. C. Bartle, A general bilinear vector integral, Studia Math. 15 (1956), 337-352.

2. J. K. Brooks, On the existence of a control measure for strongly bounded vector measures, Bull. Amer. Math. Soc. 77 (1971), 999-1001.

3. Weak compactness in the space of vector measures, Bull. Amer. Math. Soc. 78 (1972), 284-287.

4. J. K. Brooks and R. S. Jewitt, On finitely additive vector measures, Proc. Nat. Acad. Sci. U.S.A. 67 (1970), 1294-1298.

5. R. B. Darst, A direct proof of Porcelli's condition for weak convergence, Proc. Amer. Math. Soc. 17 (1966), 1094-1096.

6. J. Diestel, Applications of weak compactness and bases to vectorial integration, Rev. Roumaine Math. Pures et Appl. 18 (1973), 211-224.

7. N. Dunford and J. T. Schwartz, Linear operators. I: General theory, Pure and Appl. Math., vol. 7, Interscience, New York, 1958.

8. S. Găină, Extension of vector measures, Rev. Roumaine Math. Pures et Appl. 8 (1963), $151-154$.

9. I. Kluvanek, The extension and closure of vector measure, Vector and Operator Valued Measures and Applications (Proc. Sympos., Alta, Utah, 1972), Academic Press, New York, 1973, pp. $175-190$.

10. A. U. Kussmaul, Stochastic integration and generalized martingales, Research Notes in Math., vol. 11, Pitman, London, 1977.

11. M. Metivier, Stochastic integral and vector valued measures, Vector and Operator Valued Measures and Applications (Proc. Sympos., Alta, Utah, 1972), Academic Press, New York, 1973, pp. 283-296.

12. The stochastic integral with respect to processes with values in a reflexive Banach space, Theor. Probability Appl. 19 (1974), 758-787.

13. J. Pellaumail, Thèse, Rennes, France, 1972.

14. R. S. Phillips, On weakly compact subsets of a Banach space, Amer. J. Math. 65 (1943), 108-136.

15. P. Porcelli, Two embedding theorems with applications to weak convergence and compactness in spaces of additive type functions, J. Math. Mech. 9 (1960), 273-292.

16. M. Sion, $A$ theory of semigroup valued measures, Lecture Notes in Math., vol. 355, Springer-Verlag, Berlin and New York, 1973.

17. __ Outer measures with values in a topological group, Proc. London Math. Soc. 19 (1969), 89-106.

18. T. Traynor, S-bounded additive set functions, Vector and Operator Valued Measures and Applications (Proc. Sympos., Alta, Utah, 1972), Academic Press, New York, 1973, pp. 355-365.

Department of Mathematics, Monash University, Clayton, Victoria 3168, Australia 